\title{
COMPLETENESS PRESERVING MULTIPLIERS ${ }^{1}$
}

\section{J. S. BYRNES ${ }^{2}$ AND D. J. NEWMAN ${ }^{3}$}

Given a $\phi(x) \in L^{\infty}(-\pi, \pi)$, so that $\phi$ is a multiplier on $L^{2}(-\pi, \pi)$, it is interesting to ask when the following implication holds:

(1) If $\left\{\psi_{n}\right\}_{n=-\infty}^{\infty}$ is any complete orthonormal set (CONS) for $L^{2}$ and if $S$ is any subset of the integers, then the new set $\left\{\varphi_{n}\right\}_{-\infty}^{\infty}$ defined by $\varphi_{n}=\psi_{n}$ for $n \in S, \varphi_{n}=\phi \cdot \psi_{n}$ for $n \notin S$, is also complete in $L^{2}$.

The following theorem gives a rather simple necessary and sufficient condition for (1).

THEOREM I. (1) holds if and only if there exists a complex number $\alpha$ such that

(i) $\operatorname{Re} \alpha \phi \geqq 0$ almost everywhere (a.e.), and

(ii) either $\operatorname{Im} \alpha \phi>0$ a.e. or $\operatorname{Im} \alpha \phi<0$ a.e.

on the zero set $Z$ of $\operatorname{Re} \alpha \phi$.

Proof. Suppose first that $\left(^{*}\right)$ holds. Let $f$ be any $L^{2}$ function orthogonal to all the $\varphi_{n}$, so that

$$
\int_{-\pi}^{\pi} f(x) \psi_{n}(x)-d x=0 \text { for } n \in S \text { and }
$$

$$
\int_{-\pi}^{\pi} f(x) \bar{\alpha} \phi(x)-\psi_{n}(x)-d x=0 \text { for } n \in T
$$

(where $g(x)^{-}$denotes the complex conjugate of $g(x)$ and $T=$ comp $(S)$ ).

Now (2) says that the Fourier series of $f$ is given by

$$
f(x) \sim \sum_{n \in T} a_{n} \psi_{n}(x)
$$

and if we let the partial sum of this Fourier series be

$$
S_{N}(f, x)=\sum_{n \in T ;|n| \leqslant N} a_{n} \psi_{n}(x)
$$

we see that (3) and (4) yield

Received by the editors July 31, 1967 and, in revised form, March 21, 1968.

${ }^{1}$ Part of this was taken from the first author's doctoral thesis and was supported by the United States Steel Foundation and Yeshiva University.

2 NRC-NRL, Postdoctoral Research Associate.

${ }^{3}$ Both authors were supported by NSF grant \#GP4391. 


$$
\int_{-\pi}^{\pi} f(x) \bar{\alpha} \phi(x)-S_{N}(f, x)-d x=0, \quad N=1,2,3, \cdots
$$

By the $L^{2}$ convergence of the Fourier series we can let $N \rightarrow \infty$ in (5), and we get

$$
\int_{-\pi}^{\pi}|f(x)|^{2} \bar{\alpha} \phi(x)-d x=0
$$

Thus $f=0$ a.e. where $\operatorname{Re} \alpha \phi>0$, so that

$$
\int_{z}|f(x)|^{2} \bar{\alpha} \phi(x)-d x=\int_{z}|f(x)|^{2} \operatorname{Im} \bar{\alpha} \phi(x)-d x=0 .
$$

Combining the above with (ii) we see that $f=0$ a.e., so $\left\{\varphi_{n}\right\}$ is complete.

We now assume that $\left(^{*}\right)$ is false and thereby produce a function $\omega(x) \in L^{1}(-\pi, \pi)$ satisfying

$$
\omega(x) \geqq 0, \quad \int_{-\pi}^{\pi} \omega(x) d x=1, \quad \text { and } \quad \int_{-\pi}^{\pi} \omega(x) \phi(x) d x=0 .
$$

Indeed, suppose no such $\omega(x)$ exists. Then the linear functional $\Lambda: f \rightarrow \int_{-\pi}^{\pi} f(x) \phi(x) d x$ on $L^{1}(-\pi, \pi)$ is never 0 on the convex set $K=\left\{\omega \in L^{1}: \omega \geqq 0, \int_{-\pi}^{\pi} \omega(x) d x=1\right\}$, so that $\Lambda(K)$ is a convex subset of the plane which misses 0 . This assures the existence of a complex number $\alpha$ satisfying

$$
\begin{array}{cc}
\operatorname{Re} \alpha \int_{-\pi}^{\pi} \omega(x) \phi(x) d x \geqq 0 & \text { for every } \omega \in K \quad \text { and } \\
\alpha \int_{-\pi}^{\pi} \omega(x) \phi(x) d x \neq 0 & \text { for any } \omega \in K .
\end{array}
$$

By (9) we see that (i) holds. But (ii) must also hold, since all other cases are clearly excluded by (10). Thus $\left(^{*}\right)$ holds, and this contradiction establishes the existence of a function $\omega \in L^{1}(-\pi, \pi)$ satisfying (8).

If we now let $\left\{\psi_{n}\right\}$ be any CONS with $\psi_{0}=(\omega(x))^{1 / 2}$ and choose $T=\{0\}$ then the set $\left\{\varphi_{n}\right\}$ which is thereby generated is orthogonal to the function $(\omega(x))^{1 / 2}$. This means indeed that (1) does not hold, and completes the proof.

If we no longer consider an arbitrary CONS but restrict our attention to the standard one $\psi_{n}(x)=e^{i n x}$ our multiplier can be any $L^{2}$ function, it need not be bounded. Thus, for each $\phi \in L^{2}$, we wish to know if the following implication holds: 
(11) If we break up the integers arbitrarily into two disjoint sets $S$ and $T$, and if we let $\varphi_{n}(x)=e^{i n x}$ for $n \in S$ and $\varphi_{n}(x)=\phi(x) e^{i n x}$ for $n \in T$, then $\left\{\varphi_{n}\right\}$ is complete in $L^{2}$.

In this situation the question of necessary and sufficient conditions is left open. It appears to be a difficult one.

If $\phi(x)$ is bounded and satisfies ( $\left.{ }^{*}\right)$ for some $\alpha$ we see that Theorem I applies and (11) holds. If we assume only that $\left(^{*}\right)$ holds without requiring that $\phi$ be bounded then (11) need not hold, as the following example demonstrates.

Example (i). We define two functions $f$ and $\phi$ by

$$
f(z)=\frac{1}{(1-z)^{1 / 3}} \text { and } \phi(z)^{-}=\frac{|1-z|^{2 / 8}}{1-z} \text {. }
$$

Since $\operatorname{Re}(1 /(1-z))=\frac{1}{2}$ for $|z|=1$ we see that

$$
\operatorname{Re} \phi(z)>0 \text { a.e. for }|z|=1 \text {. }
$$

In addition, it is obvious from (12) that

$$
f \in L^{2}(-\pi, \pi) \text { and } \phi \in L^{2}(-\pi, \pi) .
$$

Furthermore, for $z=e^{i x}$ and $\bar{z}=e^{-i x}$ we have

$$
\begin{aligned}
f(z) \phi(z)^{-} & =\frac{|1-z|^{2 / 3}}{(1-z)^{4 / 3}}=\frac{(1-z)^{1 / 3}(1-\bar{z})^{1 / 3}}{(1-z)^{4 / 3}} \\
& =\frac{(1-\bar{z})^{1 / 3}}{1-1 / \bar{z}}=\frac{-\bar{z}}{(1-\bar{z})^{2 / 3}} .
\end{aligned}
$$

But (12) and (15) show that, when looked at as functions on the circle $-\pi \leqq x<\pi, f$ has no negative Fourier coefficients and $f \cdot \Phi$ has no nonnegative Fourier coefficients, i.e.,

$$
\begin{aligned}
& \int_{-\pi}^{\pi} f\left(e^{i x}\right) e^{-i n x} d x=0 \quad \text { for } n<0 \text { and } \\
& \int_{-\pi}^{\pi} f\left(e^{i x}\right) \phi\left(e^{i x}\right)-e^{-i n x} d x=0 \quad \text { for } n \geqq 0 .
\end{aligned}
$$

Thus, if we take $\varphi_{n}(x)=e^{i n x}$ for $n<0$ and $\varphi_{n}(x)=\phi\left(e^{i x}\right) e^{i n x}$ for $n \geqq 0$ we see from (13), (14) and (16) that we have a counterexample to (11).

The following theorem shows that (11) is equivalent to an interesting uniqueness property for $l_{2}$ series. 
THEOREM II. (11) is equivalent to:

(17) Suppose that $\left\{a_{n}\right\} \in l_{2}$ and $\left\{c_{n}\right\} \in l_{2}$, that $\phi(x)=\sum_{n=-\infty}^{\infty} c_{n} e^{-i n x}$ and that $a_{n}(a * c)_{n} \equiv 0$, where $(a * c)_{n}=\sum_{k=-\infty}^{\infty} a_{k} c_{n-k}$. Then $a_{n} \equiv 0$.

(Note. By equivalent we mean that (11) holds for $\phi(x)$ if and only if (17) holds for the same $\phi(x)$.)

The trivial proof, which consists of showing that a counterexample to (11) leads directly to a counterexample to (17) and viceversa, is omitted.

If we state Theorem I in terms of (17) we get

Theorem III. Let $\left\{a_{n}\right\} \in l_{2}$ and $\left\{c_{n}\right\} \in l_{2}$ and define $\phi(x)$ $=\sum_{n=-\infty}^{\infty} c_{n} e^{-i n x}$. Suppose that $\phi$ is bounded, that $\left(^{*}\right)$ holds for some $\alpha$, and that $a_{n}(a * c)_{n} \equiv 0$. Then $a_{n} \equiv 0$.

One special set of circumstances under which (17) holds is given by:

Theorem IV. Suppose that in addition to the hypotheses of (17) we also have $\left(^{*}\right)$ holds and $\left\{(a * c)_{n}\right\} \in l_{2}$. Then $a_{n} \equiv 0$.

Proof. By hypotheses $\sum_{n=-\infty}^{\infty} \bar{a}_{n}(a * c)_{n}=0$. Since $\left\{(a * c)_{n}\right\} \in l_{2}$ we can apply Parseval's formula to this equation, and we get

$$
\int_{-\pi}^{\pi}|f(x)|^{2} \phi(x) d x=0 \quad \text { where } f(x)=\sum_{n=-\infty}^{\infty} a_{n} e^{-i n x}
$$

Since $\left(^{*}\right)$ holds $(18)$ shows that $f=0$ a.e., or $a_{n} \equiv 0$.

Note that Theorem IV shows that if we have a counterexample to (11) with $\left(^{*}\right)$ holding the $L^{2}$ function $f$ which is orthogonal to all the $\varphi_{n}$ cannot be such that $f \cdot \phi \in L^{2}$.

The questions with which we dealt above can also be asked for function spaces other than $L^{2}(-\pi, \pi)$. In particular, if we are given a $\phi \in L^{p}(-\pi, \pi)$ for some $p, 1 \leqq p \leqq \infty$, we can ask whether $\left\{\varphi_{n}\right\}$ is complete in $L^{p}$, where $\varphi_{n}$ are those given in (11). With a few minor modifications the proof of the "if" half of Theorem I applies to the following result.

Theorem V. Suppose that $1 \leqq p<2$, that $\left(^{*}\right)$ holds for some $\alpha$, and that $\phi \in L^{p /(2-p)}$. Then $\left\{\varphi_{n}\right\}$ is complete in $L^{p}$.

If $1<p<\infty$ and $a$ is any number such that

$$
(p-1) / 2 p<a<(p-1) / p=1 / q,
$$

and if we define two functions $f$ and $\phi$ by

$$
f(z)=\frac{1}{(1-z)^{a}} \text { and } \phi(z)^{-}=\frac{\mid 1-z^{2 u}}{1-z} .
$$


then seen we see that, using the method of Example (i), we have a counterexample to (11) for $L^{p}(-\pi, \pi)$ with $1<p<\infty$, where $\operatorname{Re} \phi>0$ a.e. Note that if $p<2(19)$ and (20) show that the number $p /(2-p)$ given in Theorem $\mathrm{V}$ is best possible. If $p>2$ (19) assures that we can take $a>\frac{1}{2}$, so that in this case we actually do have a bounded counterexample!

Finally, we consider the space $C$ of continuous functions on the circle (i.e., continuous and periodic with period $2 \pi$ ), and we ask whether $\left\{\varphi_{n}\right\}$ given by (11) is complete in $C$.

If $\phi$ is "smooth" enough and satisfies the usual "direction property" we see by the following theorem that we have completeness.

TheOREM VI. Suppose $\phi$ is a $C^{2}$ function on $[-\pi, \pi]$ and $\operatorname{Re} \alpha \phi>0$ for some $\alpha$. Then $\left\{\varphi_{n}\right\}$ is complete in $C$.

To prove this theorem we observe that the Fourier coefficients $c_{n}$ of $\phi$ certainly satisfy $\sum_{n=-\infty}^{\infty}|n|^{1 / 2}\left|c_{n}\right|<\infty$ and then apply a result given in [1]. Since it would require the development of a new topic to even state this result we refer the reader to [1, Corollary III.2].

We conclude our work by producing a counterexample to Theorem VI when we do not assume the added restriction that $\phi \in C^{2}$.

Example (ii). We construct a function $\phi$ and a nonzero measure $d y$ such that:

$$
\begin{array}{cc}
\phi \in C \text { and } & \operatorname{Re} \phi>0, \\
\int_{-\pi}^{\pi} e^{-i n x} d y(x)=0 & \text { for } n>0 \text { and } \\
\int_{-\pi}^{\pi} \phi(x) e^{-i n x} d y(x)=0 & \text { for } n \leqq 0 .
\end{array}
$$

(22) is equivalent to

$$
d y(x)=f\left(e^{i x}\right)-d x \quad \text { where } f \in H^{1} \text { of the unit disk. }
$$

Combining (23) and (24) we get $\phi(x) f\left(e^{i x}\right)^{-}=e^{i x} g\left(e^{i x}\right)$ where $g \in H^{1}$. Thus, we want to find two functions $f$ and $g$ such that

$$
f \text { and } g \text { are in } H^{1} \text { of the disk and }
$$

$$
\phi(z)=z g(z) / \bar{f}(z) \text { is continuous and has positive real part }
$$

for $z=e^{i x},-\pi \leqq x \leqq \pi$. This is done by letting

$$
f(z)=g(z)=i(1-z)^{-1} \log ^{-3 / 2}\left(\frac{N}{1-z}\right)
$$


where $N$ is any positive number large enough so that

$$
\operatorname{Re} \log ^{3}\left(\frac{N}{1-z}\right)>0 \quad \text { for } z=e^{i x}, \quad-\pi \leqq x \leqq \pi .
$$

It is obvious that $f$ and $g$ satisfy (25), and we have

(29)

$$
\begin{aligned}
\phi(z) & =\frac{z f}{\bar{f}}=\frac{z f^{2}}{|f|^{2}}=\frac{-z(1-z)^{-2} \log ^{-3}\left(\frac{N}{1-z}\right)}{|1-z|^{-2}\left|\log \left(\frac{N}{1-z}\right)\right|^{-3}} \\
& =\frac{\log ^{-3}\left(\frac{N}{1-z}\right)}{\left|\log \left(\frac{N}{1-z}\right)\right|^{-3}} .
\end{aligned}
$$

Combining (28) and (29) we see that $\operatorname{Re} \phi>0$. Since $|\phi(z)|=1$ for $z=e^{i x}$ to show $\phi$ is continuous it is only necessary to show that it has a continuous argument. The only possible trouble could be at $x=0$, but it is clear from (28) and (29) that as $x \rightarrow 0$ from either direction $\phi\left(e^{i x}\right) \rightarrow 1$. This shows that $\phi$ satisfies (26) and completes Example (ii).

\section{REFERENCE}

1. J.S. Byrnes and D. J. Newman, Uniqueness theorems for convolution-type equations, Trans. Amer. Math Soc. 137 (1969), 383-397.

U. S. NAVAL RESEARCH LABORATORY AND Yeshiva UnIVERSITY 